\title{
Philologie de la civilisation japonaise
}

Jean-Noël Robert

\section{(2) OpenEdition}

\section{Journals}

Édition électronique

URL : https://journals.openedition.org/annuaire-cdf/15811

DOI : 10.4000/annuaire-cdf.15811

ISBN : 978-2-7226-0572-5

ISSN : 2109-9227

Éditeur

Collège de France

Édition imprimée

Date de publication : 30 décembre 2020

Pagination : 351-366

ISBN : 978-2-7226-0516-9

ISSN : 0069-5580

Référence électronique

Jean-Noël Robert, «Philologie de la civilisation japonaise », L'annuaire du Collège de France [En ligne], 118 | 2020, mis en ligne le 01 avril 2021, consulté le 22 août 2022. URL : http://

journals.openedition.org/annuaire-cdf/15811 ; DOI : https://doi.org/10.4000/annuaire-cdf.15811 


\title{
PHILOLOGIE DE LA CIVILISATION JAPONAISE
}

\author{
Jean-Noël ROBERT \\ Professeur au Collège de France
}

Mots-clés : Dôgen, philologie, civilisation japonaise, langue, poésie, bouddhisme, histoire

La série de cours «Dôgen (1200-1253) : le Zen entre deux langues » est disponible, en audio et/ou vidéo, sur le site internet du Collège de France (https://www.collegede-france.fr/site/jean-noel-robert/course-2017-2018.htm), ainsi que les colloques «Rongi : les disputations bouddhiques au Japon » (https://www.college-de-france.fr/ site/jean-noel-robert/symposium-2017-2018.htm) et "Hiéroglossie III : Persan, syroaraméen et les relations avec la langue arabe » (https://www.college-de-france.fr/site/ jean-noel-robert/symposium-2017-2018__1.htm).

\section{ENSEIGNEMENT}

\section{COURS - DÔGEN (1200-1253) : LE ZEN ENTRE DEUX LANGUES}

Il est indiscutable que l'œuvre de Dôgen, et tout spécialement le Shôbôgenzô 正法 眼蔵, ou Réceptacle de l'œil de la Loi correcte, qui a fait l'objet du cours de cette septième année, constituait l'un des sommets de notre enquête ; la raison devrait en être évidente: on trouverait en effet difficilement dans la littérature japonaise, religieuse ou non - voire dans la littérature en général avant certains romans du $\mathrm{XX}^{\mathrm{e}}$ siècle - une œuvre où la langue elle-même soit à la fois le moyen et la matière d'une véritable pratique méditative. Le Shôbôgenzô se présente avant tout comme une singulière énigme linguistique, il est en lui-même, pourrait-on dire, un «cas » de méditation, un kôan, ou kosoku. La diversité des traductions qui en ont été tentées dans les langues occidentales montre à quel point le sens nous en échappe encore. Non pas le sens littéral, ni les allusions innombrables, ni les citations textuelles qui y fourmillent. Le travail de plusieurs générations de chercheurs industrieux nous a livré, et nous livre encore, et à l'avenir aussi assurément, de précieuses indications qui ne peuvent qu'affiner notre compréhension de sa démarche textuelle, mais l'énigme demeure, une énigme si grande que l'un des meilleurs spécialistes japonais du bouddhisme chinois a pu dire un jour que Dôgen prononçait ses sermons en état 
d'ébriété. C'était bien sûr une boutade, mais une boutade qui reflète bien la perplexité de ceux qui se plongent dans la lecture du Shôbôgenzô. Cette perplexité a été aussi la nôtre au fil des années, mais une chose semble à présent assurée, au terme de ce cours, c'est que si nous espérons pouvoir suivre la démarche de Dôgen, il est indispensable de le suivre dans le va-et-vient qu'il a lui-même effectué entre les deux langues chinoise et japonaise tout au long de sa vie. Il ne s'agit pas, nous pensons l'avoir montré, d'une simple convenance littéraire liée au genre d'écrit qu'il rédigeait, mais du déroulement même de sa pensée religieuse, qui est à la fois méditation et réflexion fondée sur le processus interne de la langue, ou plutôt des langues concernées. Il est particulièrement éloquent que les deux grands recueils que nous a laissés Dôgen, les œuvres qui semblent représenter l'essentiel de sa pensée et de sa pratique, soient rédigées en deux langues (ou styles) différents, et que la dernière, l'ultime trace de sa pensée, soit en chinois, alors que ce qui a été considéré comme son chef d'œuvre était en japonais. Nous avons d'ailleurs vu que, sous l'appellation commode de «chinois » et de « japonais », se cachent en réalité des états langagiers bien plus complexes que ces noms ne donnent à penser. C'est même là que se joue tout ce qui fait l'originalité de Dôgen, une originalité qui le situe à part de tous ses successeurs. Ce n'est donc pas sans arguments que nous avons choisi de placer Dôgen dans notre enquête sur la philologie de la civilisation japonaise, et en lui accordant une place privilégiée. Mais il nous a semblé tout aussi important de partir de plus haut, de plus tôt qu'on ne le fait d'ordinaire pour tenter de reconstituer le chemin herméneutique (car c'est bien de ce terme que nous voudrions résumer son entreprise) parcouru par Dôgen. Et tout d'abord en rappelant ses origines intellectuelles.

Pour ce qui est de ses origines sociales, il y a fort peu de choses à en dire ; comme tous ceux que nous avons vu au long de ces années, qu'ils soient hommes ou femmes, Dôgen appartenait à la noblesse de l'ancienne capitale, au clan des Minamoto plus précisément. Son père putatif n'était nul autre que Minamoto no Michichika, sans que l'on sache exactement les problèmes qui ont entouré sa naissance. Sa mère était une Fujiwara, fille de Kujô Motofusa, lequel connut certes des vicissitudes politiques, mais était parvenu aux fonctions de régent (ou kanpaku) et fut le protecteur de Dôgen jusqu'à sa mort en 1230. N'insistons pas davantage sur ses origines, qui expliquent qu'il put profiter toute sa vie de relations bien établies, même après les tristes événements qui marquèrent son enfance : la mort de son père à deux ans, et la mort de sa mère à huit ans. Cela explique qu'il se tourna dès lors vers la vie religieuse, comme il était assez fréquent à l'époque. Alors qu'il résidait encore, après la mort de sa mère, dans la luxueuse villa dite Matsudono (Shôden) à Kohata, près d'Uji, auprès de son grand-père maternel, donc, il s'était déjà illustré en lisant l'Abhidharma-kośa, ce qui serait un exploit maintenant encore pour n'importe quel adulte.

\section{Dôgen et le Tendai}

Mais l'important est que dans sa onzième ou douzième année, il quitta ce lieu de rêve en des circonstances qui sont données comme quelque peu mystérieuses par sa biographie traditionnelle, puisqu'il serait parti en secret au pied du mont Hiei (cela désigne-t-il l'Onjô-ji ?) pour se consacrer vraiment à l'étude du bouddhisme sous la direction d'un prélat du Tendai du nom de Ryôkan 良観, qui était l'un de ses oncles. Le jeune garçon put alors étudier et pratiquer à Yokawa, lieu du Hiei rendu célèbre par la présence de Genshin qui y avait résidé deux siècles plus tôt. Il reçoit en 1213 la tonsure monastique et les commandements de bodhisattva du zasu de l'École, 
l'année même où Jien assura par deux fois très brièvement cette charge. Il étudia ensuite au Miidera (Onjô-ji), auprès d'un autre oncle, le préfet monacal Kôin 公糺, qui l'encouragea à s'orienter vers la pratique du Zen.

Le jeune Dôgen n'étudia donc que quelques années la scolastique Tendai, mais il le fit de façon très approfondie si nous en croyons ses biographes, qui précisent qu'il s'initia aussi à l'ésotérisme, non sans se plonger en plus dans la lecture de l'ensemble du canon bouddhique chinois, un élément important de la biographie des grands moines.

Il est à peine besoin de souligner tout ce que comporte de stéréotypé certains épisodes de cette jeunesse, avec des réminiscences évidentes de la biographie du Bouddha : le grand-père qui initie son petit-fils aux arcanes des protocoles de cour (yûsoku-kojitsu), connaissance indispensable pour qui souhaite faire une belle carrière dans l'administration impériale. L'on pourrait même voir dans l'insatisfaction du moinillon à l'égard des doctrines du Tendai une sorte de reflet de la rencontre du Bouddha avec les maîtres hérétiques (rokushi-gedô), mais ce serait peut-être aller un peu loin. Cependant, il n'est pas jusqu'au jugement d'un vieux moine à son égard qui discerne que, pourvu de la sagesse de Mañjuśrî, il est digne des facultés du grand Véhicule, il n'est pas jusqu'à ce jugement, dis-je, qui ne rappelle l'épisode du vieillard Asita dans la vie du Bouddha. Il est vrai que l'on trouverait de tels éléments dans de nombreuses vies de religieux, mais pour ceux qui ont en tête le début du Roman de Genji, antérieur de deux siècles à ces éléments biographiques concernant Dôgen, les analogies avec l'enfance du jeune prince sont indéniables. La vie du Bouddha constituait une sorte de modèle auquel on se référait sans cesse, même en dehors de la littérature religieuse proprement dite.

Quoi qu'il en puisse être du jugement des biographes du Sôtô-shû, la lignée du Zen qu'il introduisit au Japon, lesquels préfèrent passer rapidement sur l'épisode Tendai, le jeune Dôgen ne pouvait être que profondément marqué par ces années de formation. Or, qu'impliquait le fait d'avoir été formé intellectuellement dans la dogmatique du Tendai ? Contentons-nous ici d'en rappeler brièvement trois aspects qui semblent avoir imprégné en profondeur la réflexion de Dôgen et la manière dont il aborde les « dogmes » (gi) qu'il examine dans ses sermons.

1. Le Tendai a élaboré un système complet de classement hiérarchisé de l'ensemble des enseignements du bouddha, et des bouddhas, tels qu'ils sont recueillis dans le Canon bouddhique (issaikyô ou daizôkyô); ce système, appelé «classification critique des doctrines » (kyôhan) se combine avec une division chronologique des cinquante ans que dura la prédication terrestre de Śâkyamuni. Le tout, repris dans l'appellation goji-hakkyô, " cinq périodes et huit doctrines », permet d'assigner à tout «dogme », ou gi, traité par un clerc du Tendai une place précise dans les « quatre doctrines » et mène à résoudre en dernière analyse toute contradiction apparente dans la lettre des sûtras. Périodes et doctrines vont dans le même sens, puisqu'elles aboutissent à la révélation du Sûtra du Lotus (avec celui de l'Extinction suprême) et de la doctrine parfaite (engyô).

2. En corollaire de cette classification critique des doctrines, la scolastique Tendai fournit aux clercs un système exégétique très développé, avec ici aussi plusieurs grilles de lecture (nous en avons vu un exemple l'an dernier avec Jien), mais dont la structure fondamentale est la « quadruple exégèse » (shishaku 四釋), l'équivalent bouddhique extrême-oriental des «quatre sens de l'Écriture » que nous connaissons entre autres dans le christianisme médiéval. Il nous faut garder à l'esprit le quatrième niveau, «l'exégèse par contemplation de la pensée »(kanjin-shaku 観心釋), qui 
constitue une clef permettant de comprendre le développement du discours de Dôgen - fait sur lequel nous avons insisté. Ces procédés de balayage systématique des niveaux de sens, s'ils se trouvent aussi dans d'autres écoles comme le Sanron et le Shingon, sont éminemment liés au Tendai au Moyen Âge japonais, et le jeune Dôgen y a été formé très tôt et très profondément.

3. Un vieux dicton dit que le mont Hiei, le siège de l'Enryaku-ji, est caractérisé par quatre choses : le froid, l'humidité, la pauvreté, la disputation. C'est assez dire la place singulière qu'occupe dans l'imagination historique japonaise cet usage scolastique de la dispute qui a connu, dans l'école Tendai, un développement tel que l'on peut dire qu'il en est devenu le modèle pour l'ensemble du bouddhisme japonais. On parle de débat (rongi; nous n'entrerons pas ici dans les détails des classifications des diverses modalités de ces débats), ou plus exactement la "disputation», que nous définissons comme la dispute scolastique organisée à l'intérieur de l'école non pas dans le but de polémiquer avec d'autres sectes pour imposer ses vues contre les autres, mais à une fin essentiellement didactique, scolastique : elle a pour but de permettre aux clercs étudiants d'approfondir le sens des « dogmes » de l'école en les faisant passer au crible d'une controverse largement imaginaire, puisque l'objectant est en réalité d'accord avec celui qui répond. L'essor des disputations du Tendai peut être daté du $\mathrm{X}^{\mathrm{e}}$ siècle; bien que l'on ait peu de documents avant le XIV siècle, il est certain que l'un des recueils importants et anciens de cent questions disputées (Le Tendai mon.yô jizai-bô 天台問要自在房 en dix livres) est l'œuvre d'un religieux Tendai, Jômyô 静明, qui est à peu près contemporain de Dôgen, même si nous ne pouvons être certains de l'authenticité du contenu actuel. La méthode utilisée dans ces questions disputées, où les dogmes sont examinés à la fois de façon diachronique et synchronique, car les objections résument leur histoire et leur développement, tandis que les réponses les situent précisément dans l'armature scolastique définitivement établie, nous rappelle fortement la façon dont Dôgen se livre systématiquement à sa permutation combinatoire des dogmes qu'il examine et soumet à une analyse finalement dissolvante, en apparence tout au moins. Nous rappellerons que la question que nous avons prise pour exemple dans le recueil de Jômyô portait sur ce « dogme »: 《Les lettres sont libération》 (monji soku gedatsu文字即解脱). Que cette question ait été débattue à l'époque de Dôgen est un fait que nous ne pouvons ignorer.

\section{Dôgen et le Sûtra du Lotus}

Qui dit école Tendai dit assurément aussi Sûtra du Lotus. Il faut cependant rappeler que ce sûtra n'est pas l'apanage exclusif du Tendai : il est aussi fondamental dans l'école des Aspects des entités (Hossô-shî), mais l'on ne risque pas d'être démenti en affirmant que c'est le Tendai qui l'a porté à la prééminence pendant l'époque Heian, prééminence dont il n'est jamais redescendu par la suite au Japon, bien au contraire renforcée par l'apparition des courants Nichirénites, Nichiren étant luimême contemporain de Dôgen. Quelque réticence que l'on puisse avoir à admettre l'influence profonde et durable du Tendai sur Dôgen, il sera difficile de nier que le Sûtra du Lotus, le cœur scripturaire de l'École, soit resté jusqu' au bout une référence et une source de méditation pour le maître de Zen. Il se démarque en cela profondément de ses maîtres et modèles chinois d'une part et se situe aussi de façon marquante dans la lignée du bouddhisme japonais, où le Sûtra du Lotus est resté lu et pratiqué par les religieux du Zen jusqu'à l'époque d'Edo. L'œuvre principale de 
Dôgen, le Shôbôgenzô, abonde en citations explicites et implicites du Sûtra du Lotus ; les titres mêmes de plusieurs de ses sermons sont pris au Sûtra, à commencer par l'un des principaux, "L'Aspect réel des entités ", dont nous avons vu à quel point il était structuré par les dogmes Tendai, et notamment celui des « dix ainsités », jûnyoze, qui constituait le point de départ de son développement.

Le Shôbôgenzô est littéralement rythmé par les citations du Sûtra du Lotus, mais nous avons vu que l'expression poétique de Dôgen, elle aussi, témoigne explicitement de sa dévotion à l'égard de ce sûtra. Nous en trouvons des exemples précis dans ses recueils poétiques dans les deux langues. Son recueil japonais, le Sanshô-dôei 傘松 道詠 ou Les Poèmes sur la Voie composés au temple Sanshô (nom du site où se trouve l'Eihei-ji, le monastère fondé par Dôgen où il passa la dernière partie de sa vie), en particulier, mérite la plus grande attention. Malgré les doutes sérieux qui pèsent sur son authenticité en tant que recueil en raison de sa publication tardive au $\mathrm{XVIII}^{\mathrm{e}}$ siècle, le fait qu'une partie importante de la soixantaine de waka qu'il contient se retrouve dans des sources plus anciennes, et surtout la teneur même des poèmes, qui a parfois de quoi surprendre chez un adepte du Zen, donnent à penser qu'on ne peut les négliger si l'on veut retracer son univers religieux. Le recueil contient entre autres une «mini-série » de cinq poèmes sur le Lotus que pourrait reprendre sous son nom n'importe quel moine Tendai confirmé. Non seulement ils confirment ce qu'il faut bien appeler la dévotion de Dôgen envers le Sûtra, comme ce poème :

Tout au long des nuits

et les jours à pratiquer

la voie de la Loi

tout cela de ce Sûtra

est la voix et l'esprit ${ }^{1}$,

mais ils débouchent aussi sur des questions que notre moine traite en profondeur dans le Shôbôgenzô. Ainsi le cinquième poème de la mini-série :

Paysages des montagnes

échos des vallées

tous tant qu'ils sont

sont de notre Shakyamuni

la voix et la forme ${ }^{2}$.

Nous constatons que, dans ce poème, Dôgen aborde la question de la prédication de la Loi par l'inanimé (mujô-seppô 無情説法), mais qu'il le fait d'un point de vue entièrement Tendai, puisqu'il affirme très ouvertement que le poète, ou l'homme de foi qui se promène dans la nature, peut sans peine y retrouver l'écho de la doctrine du Lotus. Telle est bien la compréhension générale du dogme courante chez les moines-poètes japonais du Moyen Âge, dont le plus complet représentant est bien sûr Jien. Mais nous savons aussi, à la lecture du sermon de même titre, Mujô-seppô, dans le Shôbôgenzô, que le sens que «donne» (si l'on peut dire cela aussi simplement) Dôgen à ce dogme est fondamentalement différent de la lecture facile qu'en ont fait les poètes d'inspiration Tendai. Il est ainsi aisé de constater la profondeur de l'enracinement du maître de Zen dans le Tendai, mais nous constatons tout aussi clairement comment la hardiesse de sa pensée et l'élan que lui donne son balancement

1. Yomosugara / hinemosu ni nasu / nori no michi / mina kono kyô no / koe to kokoro to (26).

2. Mine no iro / tani no hibiki mo / mina-nagara / waga Shakamuni no / koe to sugata to (29). 
langagier, prenant appui sur une langue pour s'élever plus haut dans l'autre, et ainsi de suite, nous empêche de le décrire de façon simpliste comme une crypto-tendaïen. Il est fort possible, comme nous l'avons suggéré pendant le cours, que ces poèmes purement « lotusiens » remontent à l'adolescence de Dôgen et à ses années d'études du Tendai, et qu'ils ne reflètent que ses idées de l'époque, mais le fait demeure qu'ils ont été conservés dans un recueil censé avoir été compilé à l'extrême fin de sa vie. Que la tradition Sôtô ait tenu à préserver ces poèmes qui frappent par leur contraste avec les idées exprimées par Dôgen dans ses sermons plaident pour leur authenticité.

L'influence du Lotus ne se limite pas aux poèmes japonais de Dôgen, qui sont, il faut le rappeler, traités par les spécialistes comme appartenant au genre des « poèmes japonais à thème bouddhique », des shakkyôka. Nous l'avons aussi clairement discernée à la lecture de ses poèmes chinois, notamment avec ces deux poèmes, l'un portant sur une énigme (kôan), celle des «Trois mondes ne sont que pensée » (sangai-yuishin) :

Bien que monts et fleuves aient pour limite les nuages

c'est en vain qu'insensé on compterait de toute la terre les grains de sable

n'attendez rien des trois rangs de chutes à la Porte du Dragon

et fiez-vous à la fleur unique du Mont des Aigles ${ }^{3}$.

Et cet autre faisant partie d'une série sur le retrait du monde :

Combien me suis-je réjoui de la solitude en ma demeure de montagne grâce à elle, je lis sans trêve le Sûtra du Lotus

d'un zèle assidu, sous les arbres, qu'en est-il alors d'amour ou de haine

j'envie la voix de la pluie nocturne au profond de l'automne ${ }^{4}$,

dont nous avons remarqué qu'il s'agissait aussi d'une allusion directe à la méditation assise (zazen), mentionnée dans le même passage du Sûtra du Lotus à l'origine de ce poème.

Un dernier mot, mais important, à propos du Lotus: nous le savons bien, l'enseignement fondamental de ce sûtra porte sur "l'Aspect réel des entités » (shohô-jissô 諸法實相), auquel Dôgen consacre tout un sermon et auquel il se réfère souvent de façon directe ou indirecte. Mais ce «dogme » est en réalité beaucoup plus profondément enraciné dans la pensée de Dôgen si nous admettons, avec nombre de commentateurs, et comme nous y invite le maître lui-même dans le contexte de ses sermons, que deux termes fondamentaux de son enseignement, la «Face originelle» (honrai no menmoku 本来面目), d'une part, et «l'énigme de la présence réalisée » (genjô-kôan 現成公案), d'autre part, peuvent être tenus pour synonymes de l'Aspect réel des entités. Si nous admettons cette équivalence, c'est à l'évidence l'ensemble de la pensée bouddhique de Dôgen qui découle de ce dogme central du Sûtra du Lotus.

3. 三界唯心 : 山川縱是有雲遮, 盡地無心徒算砂, 莫待龍門三級浪, 任他熟嶺一枝華. Sansen tatoi kore kumo no saezuru ari to mo / jinji kokoro nashi, itazura ni suna wo kazoo I matsu koto nakare ryûmon sankyû no name I kano jurei no isshi no hana ni makase.

4. 山居 : 幾悅山居尤寂寞 因斯常讀法花經 專精樹下何憎愛 妬矣秋深夜雨聲 X.99. Ikubaku ka yorokobu sankyo no mottomo jakubaku taru wo / kore ni yorite tsune ni hokekyô wo yoти / senjô-juge nanzo zô.ai-sen / netamashi, aki fukaki ya.u no ne (d'après Kagamishima Genryû). 


\section{Le voyage en Chine ; Dôgen et la langue chinoise}

C'est donc ainsi armé de la scolastique Tendai, puis de la pratique du Zen qu'il se mit tout d'abord, sur les conseils de son oncle, à l'école d'Eisai au Kennin-ji, pendant six ans environ, avant de se lancer dans l'étape décisive de sa carrière de religieux bouddhiste, à savoir le voyage à la Chine. Comme on le sait, ce voyage réellement initiatique est l'étape majeure de la vie de nombre de religieux qui ont marqué profondément l'histoire du bouddhisme japonais : Saichô, Kûkai, Dôgen sont les trois noms qui viennent immédiatement à l'esprit parmi les fondateurs. On peut aussi penser aux grands docteurs Tendai du $\mathrm{IX}^{\mathrm{e}}$ siècle : Ennin et Enchin. Sans nul doute, ce voyage peut être dit initiatique au sens le plus littéral, dans la mesure où ceux qui en reviennent ont acquis par cette épreuve périlleuse une légitimité qui les met très au-dessus de leurs confrères plus sédentaires.

Mais le voyage en Chine de Dôgen se distingue même de celui de son prédécesseur immédiat, Eisai 榮西 (1141-1215), qui s'y était pourtant rendu par deux fois, en 1168 pour cinq mois à l'âge de 28 ans, et une seconde fois de 1187-1191 à l'âge de 47 ans. Il s'en distingue par deux points essentiels qui le mettent à part de tous ses prédécesseurs.

1. Tout d'abord, par cet événement on ne peut plus initiatique qu'est l'Éveil intellectif (satori) qu'il y a réalisé grâce à sa rencontre avec le maître de Chan chinois Rujing / Nyojô 如净 (1163-1228), abbé du Jingde-si / Keitoku-ji 景徳寺, dans la province du Zhejiang, en 1225, au milieu d'un séjour sur le continent qui se poursuivit de 1223 à 1227. Alors que tous ses prédécesseurs ont certes rapporté des initiations formelles, des enseignements novateurs, des rites inouïs, seul Dôgen revient «éveillé ». Nous avons vu que, dans son esprit comme dans celui de ses disciples, c'est bel et bien comme successeur de son maître chinois Rujing, comme $51^{\mathrm{e}}$ patriarche de l'école Sôtô qu'il va revenir au Japon. Par ce fait remarquable, nous voyons illustrée concrètement cette idée majeure du bouddhisme japonais : à savoir qu'il arrive une époque où l'histoire du bouddhisme chinois se poursuit plus légitimement dans l'archipel japonais que sur le continent. On peut retrouver cette idée dans l'école Tendai comme dans le Shingon, mais dans le Sôtô-shû, elle repose sur cet événement spirituel décisif qu'est le satori auquel Dôgen est persuadé d'avoir eu accès.

2. Mais l'autre aspect du voyage de Dôgen, tout aussi unique, est d'ordre linguistique. Il suffit de comparer les textes chinois d'Eisai à ceux de Dôgen pour s'en rendre compte. Il faut rappeler que tous les prédécesseurs du maître de Méditation, tous les noms que nous avons mentionnés plus haut se sont inscrits dans une unique tradition lettrée de culture de la langue chinoise classique. Les moines japonais qui se sont rendus en Chine ne parlaient probablement pas le chinois vulgaire ; on en est sûr pour Saichô (qui se fit accompagner par Gishin pour cette raison), la question est plus délicate pour Kûkai, mais on constate que le chinois parlé ne joue aucun rôle dans ses textes, pourtant exclusivement rédigés en chinois classique. Il en va de même pour Ennin et Enchin, encore que le premier ait certainement dû acquérir une connaissance pratique de la langue parlée en neuf ans de séjour (838-847), et que ce soit moins sûr pour le second avec ses cinq années (853-858). Il convient aussi de souligner que la connaissance du chinois parlé, c'està-dire de la langue vulgaire, ne jouissait pas d'une considération particulière parmi les lettrés japonais, elle était même l'indice d'un statut social inférieur en raison de son côté pratique. 
Dôgen, lui, a indéniablement acquis une connaissance opérationnelle de la langue chinoise parlée, il l'avait peut-être même apprise dès avant son départ. Cela est déjà méritant, mais ce qui effectue la rupture avec ses prédécesseurs, c'est que Dôgen entend faire de la langue si particulière du Chan chinois, dont les principaux éléments grammaticaux sont pris directement à la langue parlée des Tang puis des Song, et sont en cela radicalement différents de ceux utilisés dans la grammaire classique, commune à la Chine, au Japon, à la Corée (et au Viêtnam), la langue de ses écrits propres, la langue de ses sermons. Certes, un nombre considérable de ses textes prescriptifs en style chinois sont écrits dans la langue littéraire usuelle, mais ceux qui concernent directement sa prédication et se trouvent recueillis dans son Eihei-kôroku (voir plus bas) sont rédigés dans une imitation du style propre au Chan chinois, tout imprégné de langue chinoise vulgaire. Ce simple fait lui mériterait déjà une place de choix dans l'évolution de la connaissance et de l'usage du chinois au Japon, mais il va encore plus loin en incorporant ces éléments dans ses écrits japonais. C'est ainsi que l'on peut soutenir que le Shôbôgenzô relève du style hybride sino-japonais (wakan-konkô-bun 和漢混交文), comme on le dirait par exemple du style du Heike-monogatari, mais que les ingrédients en sont si différents que ce nouveau style est incompréhensible à ceux qui ne connaîtraient que l'ancien. Il faut d'ailleurs rappeler qu'il sera relativement peu suivi. Il suffit par exemple de lire les sermons du moine Tetsugen 鉄眼 (1630-1682) de l'école Ôbaku 黄檗宗, qui apporte pourtant des éléments novateurs dans la connaissance du chinois parlé, dont on pourrait s'attendre à ce qu'ils soient encore plus radicaux dans l'innovation langagière, pour se rendre compte du contraste dans la lisibilité des deux auteurs.

Ainsi, le séjour en Chine de Dôgen lui confère une légitimité religieuse classique, initiatique, bien qu'à un niveau encore inouï avant lui grâce à son satori, mais il est surtout l'occasion d'importer dans la langue japonaise un idiome entièrement nouveau, du point de vue stylistique, grammatical et phonétique, parfaitement identifiable et reconnaissable, et qui constituera désormais une nouvelle dimension de la hiéroglossie japonaise.

C'est d'ailleurs Dôgen lui-même qui montre l'exemple par la constitution de son œuvre ultime, l'Eihei-kôroku 永平廣録 ou Recueil étendu du temple Eihei, dont il poursuivra l'élaboration jusqu'aux toutes dernières années de sa vie. Entièrement rédigé en langue chinoise et en style Chan, recueillant des sermons de Dôgen dont on a de bonnes raisons de supposer qu'ils ont été réellement prononcés en langue chinoise, ce recueil instaure le moine japonais comme un véritable maître chinois de Chan, pleinement situé dans la lignée que reconstituera ensuite le Denkôroku 傳光 録, avec lui-même, Dôgen, comme $51^{\mathrm{e}}$ patriarche après le Chinois Rujing, et son disciple Ejô 懷焋 comme $52^{\mathrm{e}}$. La maîtrise du chinois, après l'immense détour japonais du Shôbô-genzô, montre la finalité de la pratique langagière de Dôgen comme stratégie religieuse.

\section{Dôgen et le kôan}

Bien que les recherches des quelques dernières décennies aient fait évoluer les représentations courantes, nombreux sont ceux qui distinguent encore les deux grands courants du Zen japonais, le Rinzai-shû 臨濟宗 transmis par Eisai et le Sôtôshû曹洞宗 de Dôgen, par l'accent que mettrait le premier sur la méditation du kôan ou « cas à méditer », « énigme à méditer », et le second sur la méditation assise, attitude résumée dans la formule bien connue du «Il n'y a qu'à s'asseoir », shikan 
taza 只管打坐, inlassablement répétée (et dont nous avons vu qu'elle est en partie dans le Sûtra du Lotus). Bien entendu, les choses ne sont pas si simples, et nous avons pu constater par nous-mêmes dans les textes abordés que le kôan, l'énigme, est au cœur de la pensée de Dôgen. Nous nous sommes arrêtés plus longuement sur son sermon portant sur la «Prédication de la Loi par l'inanimé » (voir plus haut), ce qui nous a permis d'apprécier le rapport langagier caractéristique de Dôgen qu'illustre ce thème. Le mouvement même de la pensée religieuse de notre moine est lancé par le va-et-vient qu'il opère entre les langues, c'est même cet élan linguistique qui le porte. Nous avons vu que le thème du mujô-seppô, l'énigme proposée, est présente à trois - voire quatre - reprises en chinois dans l'œuvre de Dôgen : il figure tout d'abord sous sa forme brute dans son Mana-Shôbôgenzô 真字正法眼蔵, le dossier de trois cents kôan qu'il s'est constitué lors de son séjour en Chine, avec les abondantes lectures qu'il a pu y faire. On ne saurait trop insister sur l'importance de ce dossier, dont la découverte est relativement récente, qui nous donne la matière première de ce que Dôgen retraitera par la suite dans les deux grandes œuvres qui ont fait l'essentiel de notre cours et qui illustre de façon idéal son parcours langagier, allant du chinois au japonais pour revenir au chinois. La même énigme est ensuite exposée sous la forme d'un véritable sermon chinois avec un poème de conclusion, poème bien sûr de langue chinoise et dans le style des poèmes d'élucidation (juko 頌 古) des kôan, mais sous deux formes différentes, au livre VI et au livre IX de l'Eiheikôroku. Et enfin (quatrième fois), il est repris dans le Kana-Shôbôgenzô (c'est-à-dire le Shôbôgenzô proprement dit) en chinois d'abord, et commenté ensuite littéralement, si l'on peut dire, en langue japonaise. Les deux œuvres majeures de Dôgen sont ainsi des élucidations de kôan, élucidations qui sont certes prélude, ou peut-être même davantage encore accompagnement, à la pratique de la méditation assise, mais qui en constitue en même temps le cadre conceptuel, et surtout linguistique : le passage qui s'opère entre l'énigme de langue chinoise et son herméneutique (qui n'est pas une exégèse explicative, nous y reviendrons très brièvement) japonaise est tout aussi fondamental pour le pratiquant que la méditation assise elle-même.

N'oublions pas, enfin, l'acception singulière que Dôgen donne au terme de kôan et qui semble lui être propre. Nous pourrions en ce sens le traduire avec raison par « énigme » ou «mystère »; c'est celui qui apparaît dans l'expression genjô-kôan, que nous pourrions presque aller jusqu'à traduire par «mystère de la présence réelle », si nous ne craignions d'être mal compris de certains. Il paraît en tout cas évident que c'est ce caractère de mystère, d'énigme linguistique à creuser par la méditation - mais la méditation qui est propre à Dôgen et qu'il déploie dans son Shôbôgenzô, méditation fondée donc sur la pratique combinatoire de la langue - que notre moine privilégie dans son intérêt profond, fondamental pour le kôan.

\section{Dôgen et la poésie chinoise}

À l'évidence, une véritable activité religieuse Chan chinoise, à l'époque où Dôgen se rend en Chine, implique aussi la maîtrise de l'expression poétique, classique bien sûr, mais aussi celle qui apparaît comme particulière aux religieux du Chan, qui fait usage de termes et de locutions de la langue parlée. On pourra assurément discuter de la pertinence de ce terme de langue parlée pour désigner des usages qui apparaissent à l'époque des Tang (voire plus tôt dans les traductions de sûtras), se poursuivent et se renouvellent sous les Song, et survivent encore dans les sermons chinois du XX $\mathrm{X}^{\mathrm{e}}$ siècle, très clairement distincts du chinois parlé contemporain (cf. les 
sermons de Xuyun). Nous pouvons préférer parler d'un idiolecte Chan, mais cela ne change rien à la question : c'est cet idiolecte que Dôgen a choisi de maîtriser et d'illustrer par sa propre création poétique, qui le met donc au niveau d'un véritable maître de Chan chinois.

Si nous reprenons l'exemple du Kôan 52 au livre IX de l'Eihei kôroku, nous voyons qu'effectivement, le poème a un but qui n'est pas que littéraire : il se présente comme une solution, certes énigmatique, à l'énigme posée :

La prédication de l'inanimé, c'est l'inanimé qui la comprend le printemps sur murs et murailles, sans parler des arbres et des herbes profanes et saints, les animés n'y ont pas part

ce sont les monts et les fleuves, le soleil et la lune, les constellations ${ }^{5}$.

Ces vers sont en réponse au débat instauré par l'énigme portant sur qui est capable de comprendre la prédication de l'inanimé, et ils expriment sous forme poétique une partie de la réponse que Dôgen fait dans son sermon japonais : le débat ne porte pas sur les profanes ou les saints, ni sur l'inanimé, car tous sont inclus dans une catégorie qui les dépasse et les subsume.

Ces poèmes sont aussi l'occasion pour Dôgen de poursuivre sa réflexion sur un même thème, même lorsque le prétexte en est à première vue indépendant. Nous avons vu par exemple comment l'anecdote de l'éveil intellectif au bruit d'un caillou cognant contre un bambou (Kôan 62) suscite chez lui cette réaction poétique :

Tout le jour, d'un cœur vide il a attendu l'arrivée du phénix mais voilà que le moine au village et l'unique sentier se sont faits voisins le dragon psalmodie, le phénix chante, il les écoute sans marquer la mesure tuiles et graviers transmettent la parole à l'homme de l'arbre mort ${ }^{6}$.

Contre toute attente, cet épisode nous ramène à la question de la prédication de l'inanimé, avec, il faut le reconnaître, une explication bien plus concrète, voire plus simple, que celle du poème explicitement consacré à la question.

Mais il faut aussi savoir parfois retrouver la langue japonaise derrière les termes pourtant bien chinois qu'utilise notre moine, avec aussi des réminiscences que l'on peut sans doute identifier, comme dans ce poème que nous avons vu aussi :

À vouloir discuter de la nature de bouddha, les deux têtes gigotent quand vent et feu se dispersent, c'est tout le corps qui frissonne naissance et mort n'ont jamais eu de sujet déterminé n'allez pas à la légère disserter de ces mots ${ }^{7}$.

\footnotetext{
5. 無情說法無情會 牆壁莫教草木春 凡聖含靈非已分 山河日月及星辰 Mujô-seppô mujô nomi e-su / shôheki (samoaraba are) sômoku no haru / bonshô-ganryô onore no bun ni arazu / sanga-nichigatsu oyobi ni seishin (d'après Kagamishima).

6. 終日虛心待鳳臻 村僧一道却成鄰 龍吟鳳唱聞無拍 瓦砂傳言枯木人

Shûjitsu kyoshin ni shite hô no itaru wo matsu / sonsô ichidô kaette tonari wo nasu / ryûginhôshô kiku ni haku nashi I garyaku (no) kotoba wo tsutau koboku no hito (ni) (d'après Kagamishima).

7. 欲論佛性兩頭動 風火散時全體寒 生死從來無定主 等閑莫說此言端

Busshô wo ron-zen to sureba ryôtô ugoku / fûka san-zuru toki zentai susamaji / shôji jûrai sadamaretaru shu nashi / naozari ni kono gondan wo toku koto nakare (d'après Kagamishima).
} 
Les deux têtes de ver de terre qui gigotent en disputant de la nature de bouddha : comment ne pas voir dans cette caricature une légère satire, pas forcément méchante, de ce qui caractérisait l'école Tendai d'où était sorti Dôgen ? Et nous rappellerons ici que le dernier mot du poème, le composé chinois gentan/gondan 言端, est une allusion fort reconnaissable et directement lisible en japonais sous la forme kotoba, « la parole », terme que nous retrouverons dans sa poésie japonaise avec un rôle considérable.

\section{Dôgen et le waka (et Jien)}

Alors que par ses écrits chinois Dôgen se situe sans ambiguïté dans la tradition des maîtres de Chan chinois (et indiens), il est malgré tout éminemment significatif que l'on ait gardé de lui un recueil, fort mince il est vrai au regard de ses poèmes chinois et au regard d'autres moines-poètes japonais, de waka qui se classent manifestement dans la catégorie des poèmes japonais à thème bouddhique (shakkyôka). Nous avons vu que ces 60 poèmes contiennent des mini-séries thématiques (le Sûtra du Lotus, la vie érémitique, les maximes du Zen). Si les synchronismes n'ont qu'une portée symbolique, ils sont malgré tout intéressants à évoquer, et l'on ne peut manquer l'occasion de souligner cette belle coïncidence : c'est en 1225, l'année où Dôgen réalisait en Chine le satori alors que son maître prononçait la formule shinjindatsuraku 身心脱落( «Dépouillement du corps et du cœur»), que disparaît le grand moine Tendai Jien, dont l'un des achèvements durables pour la littérature japonaise fut d'une part l'établissement définitif de la poésie japonaise à thème bouddhique comme genre littéraire (n'oublions pas que Jien est l'un des poètes les plus représentés dans le Shin-Kokin-waka-shû) et d'autre part la fixation de procédés littéraires permettant d'établir une correspondance systématique entre le vocabulaire poétique traditionnel et les doctrines bouddhiques. Si le modeste corpus dôgénien (de poésie japonaise s'entend) ne nous permet guère de mesurer l'influence de Jien de ce point de vue, il apparaît cependant que le grand prélat et poète du Tendai, qui fit l'objet de notre cours de l'an passé, a pu servir de modèle au maître de Zen pour certains procédés que nous avons relevé dans notre leçon sur les waka de Dôgen. Il s'agit en particulier de la présentation de poèmes bouddhiques par couples, ainsi que nous l'avons vu pour quatre poèmes à thème imposé. Ce procédé est caractéristique de Jien, nous l'avons vu notamment dans le Naniwa-hyakushu et ses cinquante couples portant chacun sur les deux vérités, et la liaison horizontale, transversale établie par Dôgen entre les intitulés des poèmes, que nous retrouvons chez Son.en, trouve sans doute chez Jien son prototype, à travers les intitulés tirés du Sûtra du Lotus par exemple, qui peuvent aussi souvent se lire transversalement.

Nous avons aussi souligné combien les waka de Dôgen paraissent à tout lecteur beaucoup plus facilement compréhensibles que ses poèmes chinois, précisément parce qu'ils se plient beaucoup plus docilement aux usages traditionnels, déjà solidement implantés par Jien. Sous réserve d'une enquête plus approfondie, nous devons bien reconnaître pour l'instant que ces poèmes se situent davantage dans la tradition bouddhique japonaise générale, voire Tendai, car rien n'empêcherait de les considérer sous cette rubrique, même ceux qui se réfèrent explicitement au Zen. Il convient de voir dans ce contraste, puisque l'on ne retrouve guère dans le KanaShôbôgenzô de parallèles doctrinaux de cet ordre - encore que certains poèmes chinois accordent, comme il l'a été souligné, une supériorité manifeste au Lotus -, une autre manifestation de la richesse de l'activité langagière de Dôgen, qui évolue 
entre ces deux pôles linguistiques chinois et japonais, dans la logique même que nous avons vue se déployer à plusieurs reprises dans les écrits de Jien.

\section{Dôgen et le koto no ha (la logique du kundoku)}

Nous avons vu que l'une des originalités de Dôgen est l'emploi substantif qu'il fait en japonais du terme chinois dô 道 dans le sens de «parole» ou «dit», directement emprunté à la langue chinoise parlée, où ce caractère se retrouve cependant essentiellement utilisé en fonction verbale, et non substantive. On relèvera que Dôgen emploie moins le terme sino-japonais monji 文字, qui peut s'entendre à la fois comme «lettre», «écriture», mais aussi «parole». Ce terme de monji se retrouve aussi bien chez Kûkai que chez Jien. Chez ce dernier auteur, on trouve bien sûr kotoba en parallèle à monji comme support de «l'acte de langage » (kotowaza) japonais par excellence qu'est le poème. Nous avons vu plus haut que le terme de monji « graphème » était associé à la libération dans une question disputée du Tendai qui pouvait remonter à l'époque de Dôgen. Mais le fait est que ce que l'on a appelé en Chine puis au Japon le monji-zen, c'est-à-dire le Zen qui repose par trop sur l'usage langagier et la rhétorique inutile, la méditation explicitée par les lettres, faisait déjà l'objet des critiques répétées des tenants d'une pratique méditative directe. Il est d'autant plus remarquable que ce soit dans le recueil de waka de Dôgen que l'on trouve à trois reprises le terme de kotoba dans sa variante poétique usuelle (koto no ha). Rappelons le premier poème :

Au neuvième mois

sur les érables

est tombée la neige

Qui donc de ceux qui la contemplent

serait dépourvu de parole ${ }^{8}$ ?

Nous pouvons ajouter le poème $\mathrm{n}^{\mathrm{o}} 53$, tout aussi éloquent en ce sens :

Dans la dispersion

de mes paroles

au vent du printemps

les gens verraient-ils

le chant des fleurs ${ }^{9}$ ?

La variante plus ancienne donnée en note n'est pas sans rappeler celle du premier poème, et elle nous amènerait à traduire : "N'y aurait-il personne / (pour dire que c'est) le chant des fleurs ? » Le poème implique, dans les deux variantes d'ailleurs, que tous ceux qui contemplent la nature y voient avant tout la prédication du Bouddha. Bien entendu, ce poème rappelle le poème sur la Face originelle (honrai no menmoku) et affirme encore une fois la véritable nature du monde qui nous entoure, le «mystère de la présence réelle ». Mais on y perçoit tout aussi bien une réaffirmation de la compréhension « ordinaire » japonaise de la prédication de la Loi par l'inanimé, dont on voit encore l'importance qu'elle a pour Dôgen.

8. Naga-tsuki no / momiji no ue ni / yuki furinu / miru hito tare ka / koto no ha no naki. Variante : uta wo yomazaran.

9. Haru-kaze ni / waga koto no ha no / chirikeru wo / hana no uta to ya / hito no miruran. Variante : hito no nakaran. 
Ces deux poèmes nous donnent aussi un aperçu profond sur ce qu'est la poésie bouddhique japonaise considérée comme une manifestation de "l'exégèse par contemplation de la pensée » (kanjin-shaku) à laquelle il a été fait allusion plus haut. Le verbe miru, «voir», signifie aussi «contempler» lorsqu'il est écrit avec le premier caractère de cette locution. Tout comme Jien, donc, Dôgen lie étroitement la poésie, la contemplation et l'exégèse disons «mystique» du Sûtra du Lotus. On rappellera que le terme pour «parole », kotoba, signifie essentiellement, s'il n'est pas précisé par un déterminant, la langue japonaise. Dôgen, en ce sens, se situe pleinement dans la lignée des poètes-exégètes japonais.

\section{Dôgen et la hiéroglossie}

Nous sommes donc parvenus, au terme d'une trop brève enquête, à l'un des sommets de ce qui a été appelé, dès le prologue de ces années de cours, la hiéroglossie japonaise, la fertilisation de la langue japonaise par la langue chinoise, sur laquelle elle prend modèle tout en s'en démarquant. Le cas particulier, et la richesse de l'œuvre de Dôgen, est que cette relation est triangulaire : chinois classique, chinois «vulgaire », japonais. Il fait intervenir dorénavant dans la culture japonaise une troisième dimension langagière qui fait de son œuvre un terrain d'enquête idéal pour le phénomène hiéroglossique.

Nous sommes encore très loin d'en avoir fait le tour. Contentons-nous de suggérer ici, pour terminer, un effet particulièrement intéressant de cette relation langagière.

Nous avons vu que l'événement marquant du séjour initiatique de Dôgen en Chine était l'intellection (satori) à laquelle il était parvenu à l'audition de la phrase shinjindatsuraku (voir plus haut), qu'on ne retrouve pas telle quelle dans les écrits Chan de l'époque. Nous avons vu aussi que l'expression était, pour ainsi dire, naturellement lue en lecture explicative japonaise, ou kundoku 訓讀, sous des formes comme $m i$ wo sute kokoro wo sutsu.

Or, on se souviendra de notre cours sur le Senjûshô que l'expression mi wo sutsu, « renoncer au corps », revenait à plusieurs reprises dans la poésie de Saigyô (mort en 1188), de même que dans les récits du Senjûshô, comme synonyme de myôri wo sutsu, "renoncer au lucre et au renom », c'est-à-dire que mi était à comprendre dans le sens de «condition sociale », et non pas de corps physique. On retrouve chez le religieux Ippen (一遍1239-1289) un poème qui mêle les deux termes :

Une fois rejeté

l'esprit qui rejeta

le rejet de ma condition

en ce monde auquel je ne pense plus

la manche teintée de mon habit de moine ${ }^{10}$

On peut donc dire qu'à l'époque de Dôgen prédominait, au Japon, l'idée du renoncement au corps «social» en même temps qu'à la pensée. Il serait alors infiniment tentant de penser que Dôgen a tout simplement mis sous un habit chinois la locution japonaise mi wo sute kokoro wo sutsu, en la sinisant par l'amalgame avec une expression qui se trouve effectivement chez Rujing (xinchen-tuoluo 心塵脱落), le fait de se débarrasser de la poussière qui recouvre l'esprit. Les nouveaux habits

10. Mi wo sutsuru / sutsuru kokoro wo / sutetsureba / omoi mo naki / yo ni sumi-zome no sode. 
chinois dont se revêt alors cette maxime, qui aurait pu effectivement le mener à l'intellection (la question n'est pas là), confère une autorité nouvelle à celui qui se veut le maître incontesté de la lignée Sôtô au Japon.

$\mathrm{Au}$ terme de cette année passée avec Dôgen, nous avons terminé sur un rapprochement : on a souvent relevé, et avec raison, le coup de génie de Matteo Ricci qui avait traduit le prologue de l'Évangile de Jean par une phrase qui s'est perpétuée jusque dans les versions modernes en chinois de la Bible ${ }^{11}$, en superposant les deux sens de dào, "voie » et "parole» pour rendre en chinois le grec logos, le verbum des Latins. Quatre siècles avant lui, Dôgen avait fait presque la même chose en incorporant dans la langue japonaise le sens chinois vulgaire « dire » ou « dit» du même caractère, dont le sens littéraire est « voie ». La version japonaise classique de Jean donne kotoba comme équivalent du chinois dào, exactement comme nous voyons chez Dôgen le dô de ses écrits chinois répondre au kotoba de ses poèmes japonais. Cette rencontre des langues par-delà les différences des religions nous donnent à penser qu'il se trouve bel et bien des convergences portées par les langues elles-mêmes, qui se concrétisent dans des situations parallèles d'adaptation, ainsi que nous le montrent Dôgen et Matteo Ricci.

\section{Colloque - Hiéroglossie III : Persan, syro-ARAméen ET LES RELATIONS AVEC LA LANGUE ARABE (25 juin 2018)}

Le colloque de cette année, le troisième de la série Hiéroglossie, était consacré au domaine persan et syro-araméen, dont les relations profondes avec la langue arabe à la suite des conquêtes musulmanes ne pouvaient être ignorées - celle-ci a été, en conséquence, incluse dans le programme. On se proposait donc d'aborder sous cet angle la «hiéroglossie », c'est à dire l'ensemble des relations langagières existant entre un groupe de langues ou entre différents états d'une même langue qui ont pour moteur principal le prestige de l'une ou de plusieurs d'entre elles, prestige fondé le plus souvent sur des raisons religieuses. La « hiéroglosse », langue sacrée ou de prestige, la langue de référence (Benoît Grévin) ou la «langue sapientielle » dont parlait Roger Bacon, devient la référence de la «laoglosse » ou le plus souvent des «laoglosses", langues de moindre prestige initial, au point que les rapports sémantiques à l'intérieur des «laoglosses » doivent être le plus souvent expliqués à l'aide des sens premiers de la « hiéroglosse ». Ainsi le japonais michi, «voie, route, chemin », ne peut être pleinement compris sans référence au chinois dào, dont c'est la lecture "explicative » japonaise (kundoku) ordinaire, le mot dào (dô en lecture "phonétique » japonaise) lui-même réunissant, outre ses sens chinois anciens, les valeurs sémantiques du sanscrit bouddhique bodhi, «Éveil, intellection », et marga, «chemin, voie », termes bien distincts en sancrit mais regroupés sous le même caractère chinois. On se reportera au résumé ci-dessus pour des exemples plus approfondis de la hiéroglossie sino-japonaise qui, rappelons-le, a servi de modèle à la lumière duquel on s'efforce d'examiner les relations langagières des grandes aires culturelles de l'Eurasie.

Pour ce troisième colloque, les communications se sont concentrées sur deux groupes de langues : d'un côté, les langues iraniennes, le persan, moyen (pahlevi) ou

11. 太初有道 太初に言あり 
moderne, mais aussi le sogdien, de l'autre, les langues araméennes, que ce soit le syriaque ou l'araméen biblique et post-biblique. La remarquable osmose qui s'est produite en moyen-persan avec l'araméen par le recours aux araméogrammes n'est pas sans rappeler, certes avec des différences considérables, l'usage que les Japonais ont fait des caractères chinois dans leurs propres langues. Cette présence de l'araméen au sein de la langue persane justifierait à elle seule le choix du thème de ce colloque, mais les relations entre les deux groupes de langues ne se limitent pas à ce système graphique. Le sogdien et le persan portèrent à travers toute l'Asie centrale des textes religieux ou philosophiques originellement syro-araméens, eux-mêmes traduits de l'hébreu par exemple, avec la Peshitta, ou du grec, avec le Diatessaron. Les rapports anciens entre le syrien et l'arabe méritent aussi d'être élucidés si l'on veut mieux comprendre les origines de l'islam.

Ce troisième colloque sur la hiéroglossie ne pouvait bien évidemment aborder qu'une infime partie des questions qui se posent. Les communications qu'il a accueillies permettent une première esquisse de ces relations langagières dont l'histoire est loin d'être terminée, puisque nous la voyons encore évoluer sous nos yeux, que ce soit avec la revivification du syriaque par la kthobonoyo ou les efforts pour redonner au pahlevi un statut de langue parlée, que ce soit directement par l'emploi de cette langue dans un contexte moderne - et l'on se doute que ces efforts ne concernent que des milieux fort limités - ou bien, à une échelle beaucoup plus visible, par le souci d'employer dans la langue moderne, où la proportion du vocabulaire d'origine arabe est comparable à celle du vocabulaire d'origine chinoise dans les langues japonaise, coréenne ou vietnamienne, des termes refaits à partir du vocabulaire médiéval.

Communications présentées :

- Jean-Noël Robert (Collège de France, Philologie de la civilisation japonaise) : «Introduction du colloque »;

- Frantz Grenet (Collège de France, Histoire et cultures de l'Asie centrale préislamique) : «Les concurrents du persan à Samarkand, $\mathrm{X}^{\mathrm{e}}-\mathrm{XI}^{\mathrm{e}}$ s., d'après les dernières découvertes »;

- Nicholas Sims-Williams (SOAS, Religions et philosophies) : «Sacred language, sacred script? Syriac and its competitors in the Christian and Manichaean texts from Turfan »;

- Arnaud Sérandour (EPHE, Section des sciences religieuses): «Le statut de l'araméen dans la Bible et la culture juive. Nouvelle proposition »;

- Muriel Debié (EPHE, Section des sciences religieuses) : «L'écriture sur la peau : de l'araméen au syriaque en ce monde et dans l'autre »;

- Christian Robin (AIBL) : «L'Arabie de Muhammad : variations dans la manière de nommer Dieu »;

- Pierre Lory (EPHE, Section des sciences religieuses) : "Pour une évaluation de la sacralité de la langue arabe en islam sunnite médiéval »;

- Bernard Heyberger (EPHE, Section des sciences religieuses) : «L'arabe et les autres langues dans le christianisme oriental $\left(\mathrm{XVII}^{\mathrm{e}}-\mathrm{XVIII}{ }^{\mathrm{e}}\right.$ siècle).

COLLOQUE - RONGI : LES DISPUTATIONS BOUDDHIQUES AU JAPON (10 octobre 2018)

Deuxième colloque HOBOGIRIN, co-organisé avec le Pr Abe Yasurô, directeur du Research Center of Cultural Heritage and Texts de l'université de Nagoya (Japon). 
- Yasurô Abe (université de Nagoya, Research Center of Cultural Heritage and Texts) : «Histoire culturelle des rongi (disputations) et des shûron (controverses). Les disputations comme matrice des textes religieux »;

- Gaétan Rappo (université de Harvard et université de Nagoya) : «Les rongi du Tendai au début de l'époque d'Edo : le moine Tenkai et les shôgun Tokugawa »;

- Paul Groner (University of Virginia) : «The encounter between song dynasty Tiantai and medieval japanese Tendai: With an emphasis on debates on the precepts »;

- Didier Davin (National Institute of Japanese Literature, Tôkyô) : «L'intrigante victoire du Zen: l'élaboration de la controverse de l'ère Shôchû et l'identité doctrinale de la branche de Daitô »;

- Kôsei Ishii (Université Komazawa): «Transformation et développement des disputations en spectacles théâtraux »;

- Kôichi Matsuo (National Museum of Japanese History) : «Les rongi-e dans le bouddhisme japonais : un rite à la base de la naissance d'une culture »;

- Jérome Ducor (Musée d'ethnographie de Genève) : «Les disputes ritualisées au Honganji »;

- Claire Brisset (université Paris Diderot): «Disputation bouddhique et parodie dans le Japon du XVI $\mathrm{XI}^{\mathrm{e}}$ siècle : à propos du "Rouleau sur les mérites comparés du saké et du riz" (Shuhanron emaki)».

\section{PuBLications}

ROBERT J.-N., « Deux traducteurs sur la Route de la Soie : traduction et réécriture du sanscrit en chinois », in S. D'InTINo et S.I. Pollock (dir.), L'Espace du sens. Approches de la philologie indienne $=$ The Space of Meaning: Approaches to Indian Philology, Paris, Collège de France, coll. «Publications de l'Institut de civilisation indienne », vol. 84, 2018, p. 451-463.

ROBERT J.-N., «L'éveil de la nature dans le bouddhisme sino-japonais : comment plantes et pierres deviennent bouddhas », in P. Descola (dir.), Les Natures en question, Paris, Odile Jacob / Collège de France, 2018, p. 33-49.

Robert J.-N., «Philologie de la civilisation japonaise », Annuaire du Collège de France 2015-2016. Résumé des cours et travaux, vol. 116, 2018, p. 351-365, DOI : 10.4000/annuairecdf.12921; en ligne : https://journals.openedition.org/annuaire-cdf/12921. 